\title{
Reflections on Plagiarism
}

\author{
Fabio Paglieri
}

Published online: 15 February 2015

(C) Springer Science+Business Media Dordrecht 2015

\section{The Facts}

It has recently come to light that an article published on this journal in 2007, "On the illuminationist approach to imaginal power: outline of a perspective", by Mahmoud Khatami, Topoi, 26(2), 221-229, extensively plagiarized parts of Mikel Dufrenne's book The phenomenology of aesthetic experience (Northwestern University Press, 1973). Entire passages from Sect. 4 of Khatami's article turned out to be copied (with minimal modifications and without acknowledgment) from chapter 11 of Dufrenne's monograph, which was not even included in the list of references. This case of plagiarism first surfaced in early November 2014 on a widely read philosophy blog, Daily Nous, in a thread of comments to a post denouncing similar alleged academic misconducts by the same author ("A case of extensive plagiarism", http://dailynous.com/2014/11/05/ a-case-of-extensive-plagiarism-guest-post/). I was first alerted of the matter a few days later, on November 15, 2014, by a fellow scholar who drew my attention to the case. After verifying the extent of the problem, I immediately informed the publisher of the journal, Springer, which in turn contacted the author of the accused article, as it is required (and rightly so) in similar instances. Since he failed to provide convincing justification for the legitimacy of his use of Prof. Dufrenne's work, it was decided to retract the article, as indicated in the retraction note available here: http://link.springer.com/article/10.1007\%

\footnotetext{
F. Paglieri $(\square)$
}

Goal-Oriented Agents Lab (GOAL), Istituto di Scienze e

Tecnologie della Cognizione (ISTC), Consiglio Nazionale delle

Ricerche (CNR), Viale S. Martino della Battaglia 44,

00185 Rome, Italy

e-mail: fabio.paglieri@istc.cnr.it
2Fs11245-015-9305-8 Therefore the article should no longer be considered as being part of that issue of the journal.

Before discussing the academic implications of this incident, which is far from isolated, I would like to express my solidarity with the relatives of the late Prof. Dufrenne and Northwestern University Press, for the unauthorized and uncredited use of his work perpetrated by Mr. Khatami, and my gratitude to those who helped revealing this sad case of plagiarism-first and foremost, Prof. Justin Weinberg at the University of South Carolina, editor of Daily Nous and author of the post where the matter first came to light. Their vigilance was a virtuous application of the "many eyes principle", and a valuable service to the whole profession. Moreover, having discussed the matter with them, I can attest that this sentiment is shared also by Prof. Ermanno Bencivenga, founding editor and editor-inchief of Topoi in 2007, and Prof. Hajj Muhammad Legenhausen, guest editor of the issue where the incriminated article appeared.

\section{The Problem with Plagiarism: Tarnished Originality or Rule Violation?}

Besides making amends and giving credit where credit is due, I believe we should take this opportunity to reflect on the negative impact of plagiarism ${ }^{1}$ on academic practices, and how we can ensure it is dealt with effectively. In order to do that, we must first neutralize a common

\footnotetext{
${ }^{1}$ In this editorial, "plagiarism" refers only to the illicit appropriation of work done by others, without proper reference. Thus I am not interested to discuss here self-plagiarism. This should not be taken to imply any benevolent inclination towards self-plagiarism; yet it is a different matter, one that I do not intend to discuss now.
} 
misconception, one that often derails the debate on plagiarism in unproductive directions: the idea that plagiarism is problematic because it violates the absolute virtue of originality. This assumption immediately raises a host of objections against the value of the very notion of "originality", while it distracts from the real problem with plagiarism: its blatant and malicious violations of the shared rules of the academic game. The point was made most effectively in 2010 by Stanley Fish in an op-ed on The New York Times, ${ }^{2}$ so I am going to quote him extensively here (hopefully, without plagiarizing him):

Plagiarism is (...) an insider's obsession. If you're a professional journalist, or an academic historian, or a philosopher, or a social scientist or a scientist, the game you play for a living is underwritten by the assumed value of originality and failure properly to credit the work of others is a big and obvious no-no. (...) Now if plagiarism is an idea that makes sense only in the precincts of certain specialized practices and is not a normative philosophical notion, inquiries into its philosophical underpinnings are of no practical interest or import. In recent years there have been a number of assaults on the notion of originality, issuing from fields as diverse as literary theory, history, cultural studies, philosophy, anthropology, Internet studies. Single authorship, we have been told, is a recent invention of a bourgeois culture obsessed with individualism, individual rights and the myth of progress. All texts are palimpsests of earlier texts; there's been nothing new under the sun since Plato and Aristotle and they weren't new either; everything belong to everybody. In earlier periods works of art were produced in workshops by teams; the master artisan may have signed them, but they were communal products. In some cultures, even contemporary ones, the imitation of standard models is valued more than work that sets out to be path-breaking. (...) $[\mathrm{I}] \mathrm{t}$ has seemed to many that there is a direct path from this line of reasoning to the conclusion that plagiarism is an incoherent, even impossible, concept and that a writer or artist accused of plagiarism is being faulted for doing something that cannot be avoided. (...) That might be true or at least plausible if, in order to have a basis, plagiarism would have to stand on some philosophical ground. But the ground plagiarism stands on is more mundane and firm; it is the ground of disciplinary practices and of the histories that have conferred on those practices a strong, even undoubted (though revisable) sense of what kind of

\footnotetext{
2 "Plagiarism is not a big moral deal", New York Times, August 9, 2010, http://opinionator.blogs.nytimes.com/2010/08/09/plagiarism-isnot-a-big-moral-deal/.
}

work can be appropriately done and what kind of behavior cannot be tolerated. If it is wrong to plagiarize in some context of practice, it is not because the idea of originality has been affirmed by deep philosophical reasoning, but because the ensemble of activities that take place in the practice would be unintelligible if the possibility of being original were not presupposed. And if there should emerge a powerful philosophical argument saying there's no such thing as originality, its emergence needn't alter or even bother for a second a practice that can only get started if originality is assumed as a baseline.

I am in full agreement with Fish's position, ${ }^{3}$ with two qualifications. First, discussing the philosophical underpinnings of originality can have a lot of practical value, certainly not for assessing current cases of plagiarism, but rather for considering whether the rules demanding a commitment to originality in academia are truly conducive of its legitimate goals, i.e. the fostering and sharing of knowledge. Obviously, even if we were to agree (I don't) that those goals are not best served by a commitment to originality, that would do nothing to alleviate the severity of previous violations of that particular norm, but it would strongly suggest we need to change the rules. Second, while I agree with Fish that plagiarism is to be condemned as a breach of conduct rather than as a moral sin per se, the act of knowingly and covertly breaking a shared rule after agreeing to obey it is in turn an obvious moral sin. So, on my view, plagiarism is not an amoral failing, as Fish would have it, but rather a derivatively immoral act-it is morally wrong not because originality is a virtue, but because cheating is a vice. ${ }^{4}$

Regardless these minor differences, Fish and I still endorse the same basic suggestion, when it comes to debating plagiarism: if we want to get to the bottom of what's wrong with it, we must quit blabbing about the pros and cons of originality, and discuss instead its real nature-a breach of some basic rules in the game of knowledge.

\footnotetext{
3 At the time when it was published, Fish's op-ed engendered a fair amount of equivocation, since many (superficial) commentators took it to imply that plagiarism was no big deal—which is certainly not his point. This prompted Fish himself to further discuss the matter, here: http://opinionator.blogs.nytimes.com/2010/08/16/the-ontology-of-pla giarism-part-two/ ("The ontology of plagiarism: part two", New York Times, August 16, 2010).

${ }^{4}$ Another way of putting a similar sentiment is to argue in favor of harsh punishments for plagiarists from an expressivist position on punishment: by recommending firm measures against plagiarism, the academic community expresses a commitment to norms aimed at promoting honesty. This stance has ethical value, regardless of its effectiveness in deterring plagiarists. The fight against plagiarism is ultimately a quest for honesty, not originality. I am grateful to Prof. Hajj Muhammad Legenhausen for helping me articulating this point in private correspondence.
} 


\section{Laying Blame and Feeling Good}

Since plagiarism is bad, when it happens we naturally start looking for a culprit, someone to blame and upon whom to exact just retribution. Those who perpetrated the offense are, quite naturally, the first target of our indignation: as I shall discuss later on, this is actually a good instinct, not because it satisfies our vindictive nature, but because it helps in keeping the system honest. Unfortunately, blame throwers are famously inaccurate in selecting their targets, so it is often the case that other parties end up smeared in the process: reviewers, editors, journals, publishers, even academia as a whole. In all these cases, the basic reasoning, tinted by a satisfying feeling of outrage and superiority, is always the same: "How could they let it happen?!"

In hindsight, plagiarism is a manifest sin. Take Khatami's article as a case in point: Table 1 compares an excerpt of it with the corresponding, plagiarized passage in Dufrenne's book. Seen next to each other, the similarity is absolutely obvious, and only an idiot would miss it—hence the hasty conclusion that those responsible for ensuring the integrity of journal articles (reviewers, editors, publishers, academics in general) must be idiots or, even worse, in league with the plagiarist. But of course the crucial information, i.e., where to look, is available only in hindsight, after the fact has been discovered and appropriate bibliographical evidence unearthed. To which, a sceptic would reply that nowadays there are plenty of software programmes designed to detect plagiarism, so extensively checking a new manuscript against previous writings is no longer as impossible as it was before the digital era. So, if scholars do have available resources to perform the required checks, then why do we still have a relative abundance of published plagiarisms, and why does it take so long to detect them?

There are two answers to this question, one weak and one strong. The weak answer is to note that the reliability of automatic plagiarism detection is far from perfect, ${ }^{5}$ since not everything is appropriately digitized and thus accessible to these programmes, ways of gaming the system are being invented as we speak, and checking every single sentence of every new manuscript against all previously published works would still be incredibly tedious and timeconsuming, given that the process is only semi-automatic (software programmes detect similarities between texts, but cannot discriminate between legitimate citations and

\footnotetext{
5 Readers interested in plagiarism detection programmes will find a list here: http://elearningindustry.com/top-10-free-plagiarism-detec tion-tools-for-teachers. Plagiarists interested to avoid getting caught will find tricks to bypass detection here: http://scholarlyoa.com/2013/ 02/07/five-ways-to-defeat-automated-plagiarism-detection/. As always in similar instances, the arms race goes on, and the only sure outcome is a proliferation of nasty practices on both sides.
}

plagiarisms). This answer is perfectly veridical, yet weak nonetheless, because vulnerable to further objection, along the following lines: "Yeah, thoroughly checking for plagiarism is hard work. So what? It's part of your responsibility as a [insert here your favorite target], so stop whining and learn to do your job!"

This leads me to the second, stronger answer, which is precisely to deny that the obsessive policing of potential plagiarism is part of the required vigilance among academics. In fact, not only it is not required, it is not even desirable. To understand why, we must attend to the social nature of academic practice.

\section{The Value of Trust and the Role of Punishment}

What is worst in plagiarism is not its utter lack of originality, nor the violation of the authors' right to be properly acknowledged, although that's bad enough; the worst is the breach in the mutual trust academics have (and should continue to have) in each other. All scholars want peer reviewing to be as rigorous as possible, but they also know very well, as reviewers of an increasing number of papers, that their resources are limited. Thus we want them to be free of focusing their attention on matters of content, not on policing sentence by sentence whether the author is plagiarizing someone else-where "someone else" could be any published material in history, no less. Precisely because we want reviewers to be manically methodical and precise in assessing the merits of an article, we need them to be able to take some basic facts for granted: proper acknowledgment of quoted sources is one of these facts.

This, of course, creates a vulnerability: trust is needed for the system to work, and plagiarists abuse this trust. Nor is this an isolated or unfamiliar instance. The collective construction of knowledge is a public good, and as any public good it is vulnerable to cheaters. Cheater-detection mechanisms are in place, but they are far from perfect, and necessarily so. So we must complement ex ante cheaterdetection with ex post punishment of cheaters, once their misdeeds come to light. Punishment, in this context, should not be understood as a morally justified sanction against some original sin, but rather as a necessary incentive to ensure the smooth functioning of a system worth preserving. This is indeed a consequence of the very nature of plagiarism, as discussed before: it is an attack against the shared rules of academic practice, rather than an assault against the absolute value of originality.

The particular function of punishment in respect to plagiarism justifies two features it must possess, in order to be effective: non-proportionality and publicity. The punishment of plagiarism is not proportional to the crime, nor should it be; in fact, it must be designed to be extreme in all 
Table 1 One of the plagiarized passages in Khatami's article, next to the original text by Dufrenne

\begin{tabular}{|c|c|}
\hline Dufrenne, 1973, p. 336 & Khatami, 2007, p. 225 \\
\hline $\begin{array}{l}\text { Consequently, a theory of meaning must begin by describing an } \\
\text { existential plane of perception in which presence to the world is } \\
\text { realized and in which there is manifested an ability to read directly } \\
\text { the meaning borne by the object- that is, in living it without having } \\
\text { to decipher or explicate a duality. This theory should beware of the } \\
\text { convenient and dangerous notion of "representation" which results } \\
\text { from the notion of a closed consciousness. To penetrate the palace of } \\
\text { consciousness, the thing would have to undergo a metamorphosis in } \\
\text { order to be presented to us. Representation would then be an event } \\
\text { which occurs inside one's mind and to which the object is admitted- } \\
\text { like a private spectacle within closed doors which consciousness } \\
\text { furnishes to itself with the means at hand, namely, the images } \\
\text { registered by memory and stored in the unconscious, and the innate } \\
\text { ideas which are also interior to the mind }\end{array}$ & $\begin{array}{l}\text { Consequently, from a Sadraean point of view, a theory of meaning } \\
\text { must begin by describing an existential plane of the imaginal faculty } \\
\text { by which presence to the world is realized reflectively and in which } \\
\text { there is manifested an ability to read directly the meaning borne by } \\
\text { the object-that is, in living it without having to decipher or explicate } \\
\text { a duality. This theory should beware of the convenient and dangerous } \\
\text { notion of "representation," (tamassul), which results from the notion } \\
\text { of a closed consciousness. To penetrate the palace of consciousness, } \\
\text { the thing would have to undergo a metamorphosis in order to be } \\
\text { presented to us. Representation would then be an event which occurs } \\
\text { inside the subject and to which the object is admitted-like a private } \\
\text { spectacle within closed doors which consciousness furnishes to itself } \\
\text { with the means at hand, namely, the images registered by memory } \\
\text { and stored in the unconscious, and the innate ideas which are also } \\
\text { interior to the subject }\end{array}$ \\
\hline
\end{tabular}

cases where the accusation of plagiarism can be satisfactorily proven, regardless of contextual factors that may be invoked as extenuating circumstances. Plagiarists have to pay always the full price of their misdeeds, no matter whether they are first-strikers or serial offenders, newbies barely acquainted with the ropes of scholarship or hardened professionals that plagiarized in cold blood. The penalty must always be as harsh as possible. ${ }^{6}$ The reason for this is simple: punishing a plagiarist is not intended to sanction a past transgression (in which case proportionality would be an obvious moral obligation), but rather to avoid future outbreaks of the same malaise. Sanctioning plagiarism is not about getting even on moral grounds, it's about building a better academia to live in. Thus the punished plagiarist has to serve as a cautionary tale for anyone else who may be tempted to follow the same path.

This is the reason why such punishment needs also to be entirely public, and the motivation for this editorial: the retraction of a plagiarized article should never be a sordid affair to hush up, but rather a public event that attracts as much attention as possible. It is a civil rite to be consumed in the agora, not behind closed doors. It is not enough to cause maximum harm to the plagiarists; everybody must also know that this is what happens to plagiarists, once caught. The publicity of the punishment is crucial for three distinct reasons: (1) it contributes to maximize the penalty, ruining the culprit's reputation; (2) it is necessary to ensure the desired deterrent effect; (3) it provides justification for the harshness of future similar punishments, by making sure that all interested parties are fully aware of what they

\footnotetext{
${ }^{6}$ Refusing to weigh extenuating circumstances should not prevent us from considering aggravating ones, though: while being young and foolish is no excuse for plagiarism, trying to get away with plagiarism thanks to a position of academic power, or repeatedly committing the offense over one's career are certainly good reasons to make sure the punishment is, in these cases, even harsher.
}

risk when they decide to plagiarize. The latter concern is becoming increasingly important, since there is mounting evidence that younger generations have troubles understanding the very notions at the core of academic plagiarism (originality, intellectual property, source crediting, etc.), ${ }^{7}$ and thus are increasingly likely to err by mistakewhich is something we generally must not condone, lest it becomes an easily invoked excuse. Yet, even the wellknown legal principle ignorantia juris non excusat applies under the assumption that the law in question has been properly communicated to those subject to it, and the same applies to the rules of the academic game. All the more reason to flog condemned plagiarists in the public eye-so to speak.

Needless to say, the very destructive and irreversible nature of the punishment demands that the charge of plagiarism is not wielded lightly: a strong presumption of innocence and high standards of proof must be firmly in place. This is why I entirely endorse the thorough and cautious process by which the publisher acted in this instance, contacting the alleged plagiarist and carefully weighing his arguments. In contrast, I do not approve the widespread tendency to conduct these matters unilaterally and in haste: while online forums can be extremely helpful in detecting suspicious papers and potentially questionable practices, as it happened in this very case, these matters need to be resolved elsewhere, and with the utmost care. Otherwise, in this as in many other matters, the wisdom of the crowd quickly turns into the frenzy of the pack.

\footnotetext{
7 This development is well documented in Susan Blum's monograph, My word! Plagiarism and college culture (Cornell University Press, 2009), although I do not necessarily share her interpretation of it. A slimmer, yet informative discussion of the same phenomenon is provided by Trip Gabriel's piece in the New York Times (August 1, 2010), "Plagiarism lines blur for students in Digital Age", http:// www.nytimes.com/2010/08/02/education/02cheat.html.
} 


\section{House Rules}

In light of these considerations, I would like to inform readers, authors, reviewers, members of the editorial board, and all other interested parties that the following rules on plagiarism apply to this journal-as I suspect and hope they do, explicitly or implicitly, in many other academic outlets:

- Harsh penalties Those who are caught in acts of blatant plagiarism will have their submission either rejected without further justification (if they are caught prior to publication) or promptly retracted (if the plagiarism is identified later on, as it happened in this case). In addition to that, their work will no longer be welcome to Topoi, as long as I am editor-in-chief: all their subsequent submissions will be rejected without review, regardless of whether they contain further plagiarism, and with no consideration for any other value they might have. In short, if we catch you plagiarizing, we will banish you forever from this journal. Moreover, I will personally take steps to spread this ostracism as widely as possible: for instance, I have already suggested the same measures to Springer, regarding all their publications, in relation to the case of Mr. Khatami. Whether or not this suggestion is accepted depends entirely on the publisher, and I suspect it won't be, due to legal considerations. But there are other ways of blacklisting plagiarists, e.g. online publicity, and these will be strenuously pursued.

- No blame sharing In contrast, this journal will never blame cases of undetected plagiarism on reviewers or editors of the articles in question. As far as we are concerned, the only culprit of plagiarism are the plagiarists themselves; everybody else is a damaged party. A corollary of this policy is that we will not ask reviewers to become "plagiarism sleuths", whereas of course we will demand from them careful scrutiny of submitted articles on all relevant aspects. As explained, it is precisely to allow reviewers to maximize their efforts on what matters that we do not want them to obsess on potential plagiarism. In addition, turning peer-reviewing into some sort of originality patrolling would erode the basic trust academics should have in each other, thus making plagiarism more damaging than it is already.

- Broadcasting the message The retraction of articles due to plagiarism will be a very public affair, witness this editorial. Moreover, even attempted plagiarism will be publicly denounced, once discovered: if your submission turns out to contain plagiarism, not only it will be rejected and you will become persona non grata to our journal; we will also do our best to alert the whole academic community of the fraud you tried to commit, so that others can either refuse to deal with you, or at least approach such dealings with caution.

- Due process and fair trial Given the apocalyptic nature of the penalties for plagiarism, we intend to make everything in our powers to ensure no innocent has to suffer them. Luckily, plagiarism is a fairly objective failing, once conceived as a breach of contract in a mutually agreed practice, so that considerations on the true nature and value of originality do not affect its detection. Nevertheless, the textual evidence will be carefully reviewed before even raising a charge of plagiarism, and the accused will always be given ample opportunity to explain themselves and, whenever appropriate, to rectify any unintentional blunderassuming there are reasons enough to consider it truly unintentional, that is. This is exactly what happened in Mr. Khatami's case.

So, please take notice. These are the rules currently in effect here at Topoi: if you publish with us, play by them, or be ready to pay the price-whatever your beliefs are on the value of originality. 